\title{
Crystallography for University Research: Some Basic Case Studies
}

\author{
Aurelien Crochet $^{\star}$
}

\begin{abstract}
An overview of commonly available, state-of-the-art diffraction techniques for university research is presented based on some chemical case studies. Examples from the Fribourg crystallography service are presented, aimed at scientists who would like to learn about the principal possibilities that X-ray powder and single-crystal diffraction can offer, how these methods can serve to determine the structure of a given material, and how they can help to identify polymorphs, surface coatings and enantiomers.
\end{abstract}

Keywords: Coatings $\cdot$ Powder diffraction $\cdot$ Single crystals $\cdot$ X-ray crystallography

One of the major issues in inorganic, organometallic or organic chemistry research is the characterization of (mostly new) products. Traditionally, elemental analysis, mass spectrometry (MS), infrared (IR) and UV/Vis spectroscopy as well nuclear magnetic resonance (NMR) spectroscopy are used to analyze molecular reaction products, but a panoply of complementary analytical techniques exists for solid-state compounds, such as X-ray photoelectron spectrometry (XPS), scanning electron microscopy (SEM) or transmission electron microscopy (TEM). Especially diffraction techniques provide many possibilities to characterize solid-state materials.

The first X-ray diffraction experiment is attributed to Max von Laue who in 1912 exposed a crystal of copper sulphate to an X-ray beam and discovered a diffraction pattern on a photographic plate placed behind the crystal. ${ }^{[1]}$ Albert Einstein commented this experiment as "one of the most beautiful" that physics had seen at that time. The first structure solved by W. L. Bragg ${ }^{[2]}$ and his father W. H. Bragg in 1914 at atomic level by X-ray diffraction is the one of sodium chloride, proving the existence of ionic compounds. In the same year the structure of diamond was established. [3] Up to now, some 28 Nobel Prizes in physics, chemistry and medicine/ physiology have been related to crystallography, the first one in physics going to W. Röntgen in 1901 for the discovery of

\footnotetext{
${ }^{\star}$ Correspondence: Dr. A. Crochet

University of Fribourg

Fribourg Center for Nanomaterials (FriMat)

Department of Chemistry

Chemin du Musée 9

$\mathrm{CH}-1700$ Fribourg

Tel.: +4126300 9132

E-mail: aurelien.crochet@unifr.ch
}

X-rays and one of the most recent ones in chemistry to D. Shechtman in 2011 for the discovery of quasicrystals.

In November 2013, the term 'crystal structure' as publication topic can be found ca. 30'500 times in publications of the Web of Science, while in 1992, the records show around 10'700 hits. ${ }^{[4]}$ This enormous increase of the number of publications dealing with crystal structures shows that diffraction techniques are more and more commonly used in research. This is for one part due to the widespread use of laboratory X-ray diffraction systems but also to the wide range of samples that can be investigated with this method: single crystals, ${ }^{[5]}$ powders, ${ }^{[6]}$ coatings $s^{[7,8]}$ and even crystalline polymers. ${ }^{[8,9]}$ The choice of the technique(s) used depends on the sample type and the desired (or expected) information. Although modern programs make structure resolution look simple, it nevertheless requires know-how and experience to solve a structure properly, especially if the structure is complicated and complex. Many scientists thus turn to the help of a crystallography service. The locations of such service units in Swiss universities can be found on the website of the Swiss Society for Crystallography (SGK-SSCr, website: http://www.sgk-sscr.ch/).

In order to illustrate the potential of diffraction methods available in many university service laboratories, some examples are given in the following. All these experiments were performed with laboratory X-ray equipment. More powerful sources like synchrotron radiation are also available in Switzerland, namely at the Paul Scherrer Institute (PSI).

\section{Powder Diffraction}

Phase identification of crystalline powders is the most basic information that can be gained by powder diffraction. The measured pattern is compared with either the theoretical diffractogram derived from single crystal data or with powder patterns of a database, such as the Crystal Structure Database, ${ }^{[10]}$ Crystal Open Database, ${ }^{[11]}$ Inorganic Crystal Structure database, ${ }^{[12]}$ or the American Mineralogist Crystal Structure Database. ${ }^{[13]}$ If no reference diffractogram is available, this method can at least determine whether the starting material has reacted to give a new product.

A more sophisticated approach to powder data is to determine crystallographic parameters from such a sample using Rietveld refinement. In some cases, just the lattice parameters are required, while in other cases, determination of symmetry can also be useful. ${ }^{[14]}$ For instance, zeolites are materials used in many fields such as chemistry, nuclear physics, construction or animal care, and a special database of the structures of these crystalline compounds is available, visualizing their channel systems. ${ }^{[15]}$ Extremely well defined in the literature and easily prepared in different shapes and sizes, two types of zeolites L were prepared in the group of P. Belser at the University of Fribourg, one forming sticks/rods (sample $S$ ) and the other one in disk shape (sample D). ${ }^{[16]}$ In order to verify the size and shape of the obtained nanoparticles, the two batches were analyzed with SEM and investigated in transmission mode with a powder diffractometer (Fig. $1)$.

At first sight, both powder diffractograms of the two samples clearly identify Zeolite L with only minor differences in the unit cell dimensions and the crystallite size, which can be derived from the peak broadness at half the maximum intensity, using the Scherrer equation (Fig. 1, Table 1). However, a difference in the presence and intensity of diffraction peaks corresponding to the planes (101) and (300) be- 


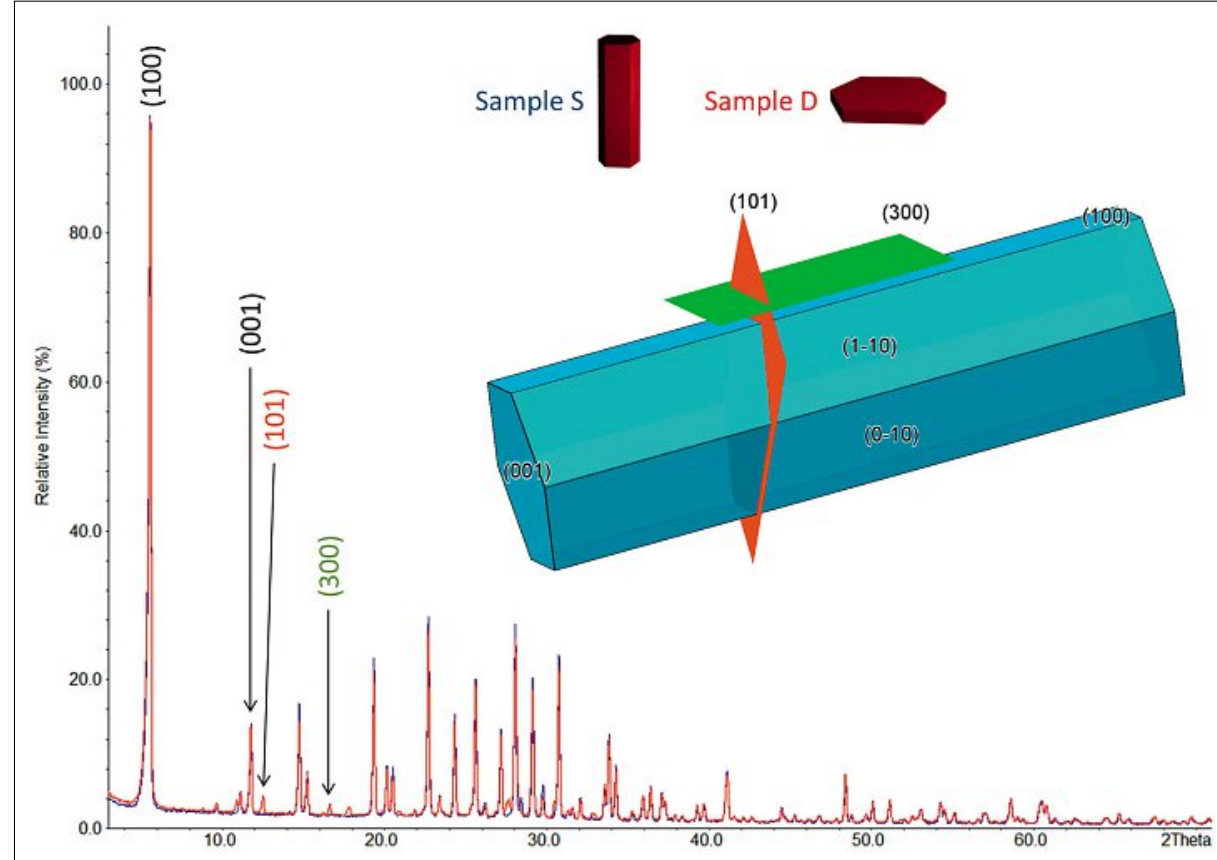

Fig. 1. Comparison of diffractograms obtained from two zeolite $L$ samples.

Table 1: Unit cells obtain for sample S and D.

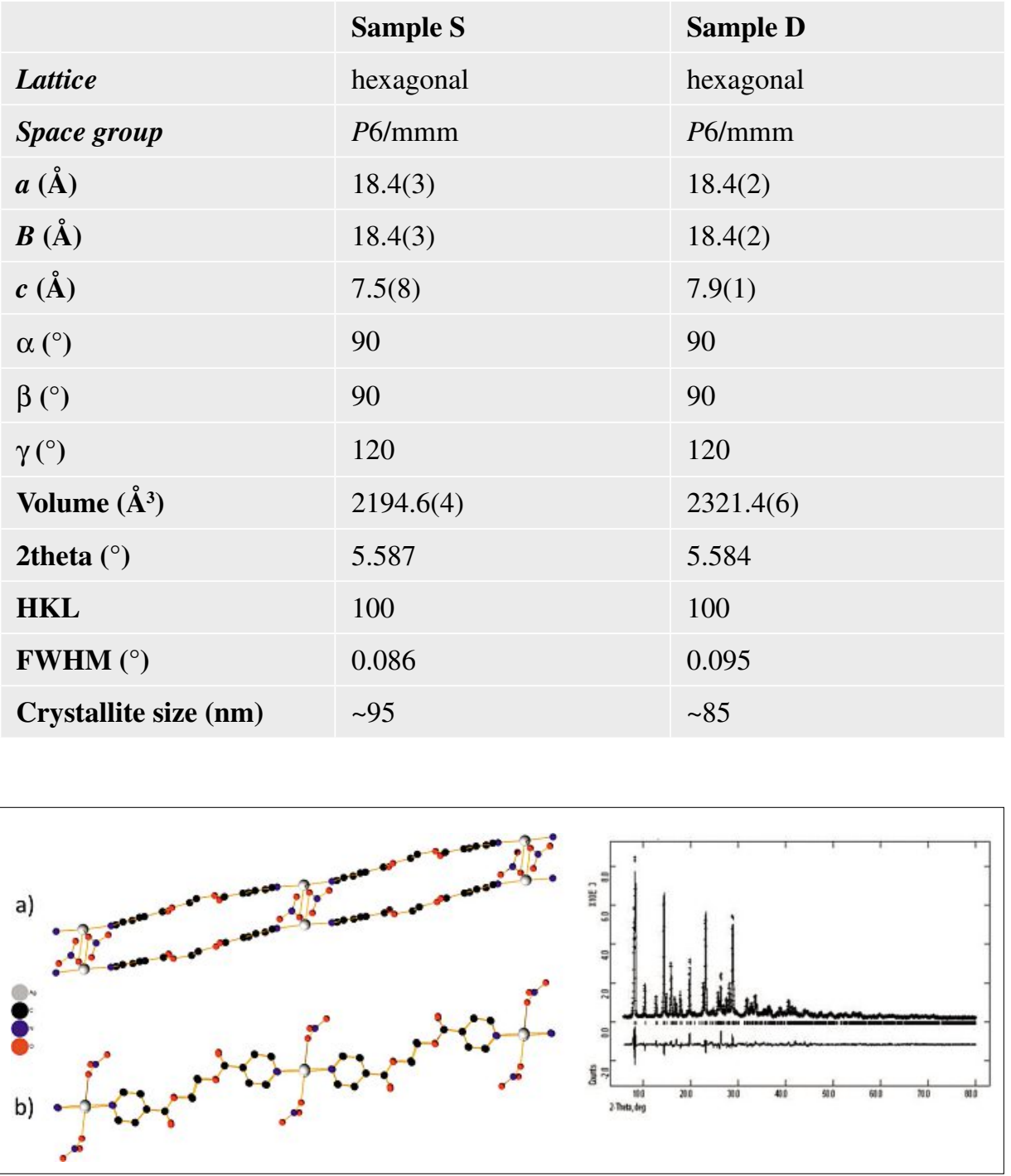

Fig. 2. Chain motifs (a) top-view of a double chain; (b) side-view of a double-chain (left); diffractogram and fitting obtained from the powder structure resolution (right). came apparent. These diffraction peaks do not correspond to major diffraction peaks and their presence reveals a frequent occurrence for this plane, as is the case in sample D. These peaks can also be observed in sample $\mathrm{S}$, but they are very small compared to the ones in sample D. This difference is due to the shape of the crystals, long sticks/rods versus large discs, as illustrated in Fig. 1.

Structure resolution is the Holy Grail of crystallography, mostly performed on a single crystal. In some cases, the single crystals are too small for single crystal diffraction on classical laboratory equipment or are simply impossible to obtain. In these cases structure resolution of the powder diffraction data can be a solution. Such a case occurred in the group of K. Fromm (University of Fribourg) for a set of silver coordination polymers prepared for their antimicrobial properties. By mixing silver nitrate with the ligand ethanediyl bis(isonicotinate) in different solvents, five different compounds were obtained.[17] Four of them crystallized as single crystals and represented two solvent-free compounds and two solvated silver coordination polymers. The fifth compound could only be obtained as a crystalline powder with a diffractogram different from the simulated patterns of the four identified compounds. By using the structural information obtained from the four known structures on the ligand as model input for the structure solution, the unknown compound (Fig. 2) could finally be identified with the help of A. Neels. ${ }^{[17]}$

Since these silver coordination polymers showed good biocompatibility and antimicrobial properties, ${ }^{[18,19]}$ they were tested as implant coatings for the prevention of implant infection and therefore coated on $\mathrm{Au}(111)$ and titanium surfaces (Fig. 3, Fig. 4).

For medical purposes, it is crucial to identify which of the above-mentioned compounds is present on the gold or titanium surface. Phase identification was thus carried out by powder X-ray diffraction measurements of the coated surfaces using the reflection mode. Since the coating is very thin (at the nanoscale), the main diffraction peak is the one generated by the $\mathrm{Au}(111)$ surface in the case of the gold substrate. Yet, zooming between $4^{\circ}$ and $25^{\circ}$ $2 \theta$, the observed diffraction peaks allow a comparison with the theoretical peaks of the possible compounds and thus the identification of the compound attached to the surface (Fig. 5). In case of product mixtures, the relative ratio between the peaks will give an indication as to how much of each product is formed.

Such a method is thus useful in materials science for thin layers and is crucial in quality control in industry. [20] 


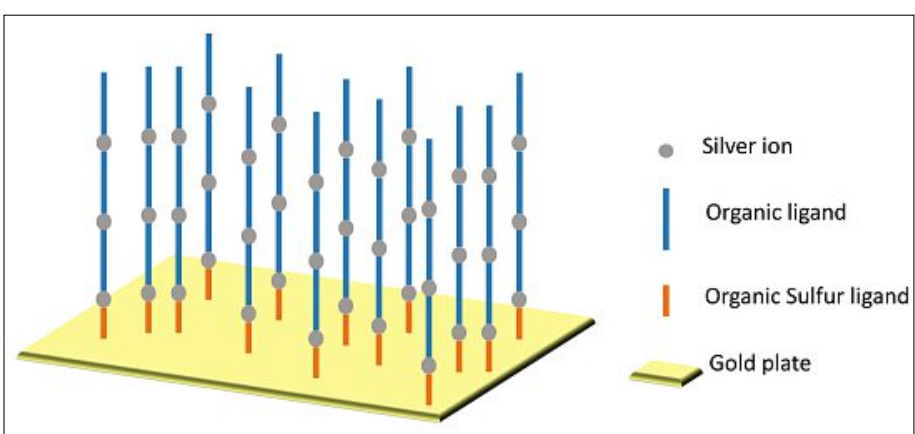

Fig. 3. Model of coating on gold plate.

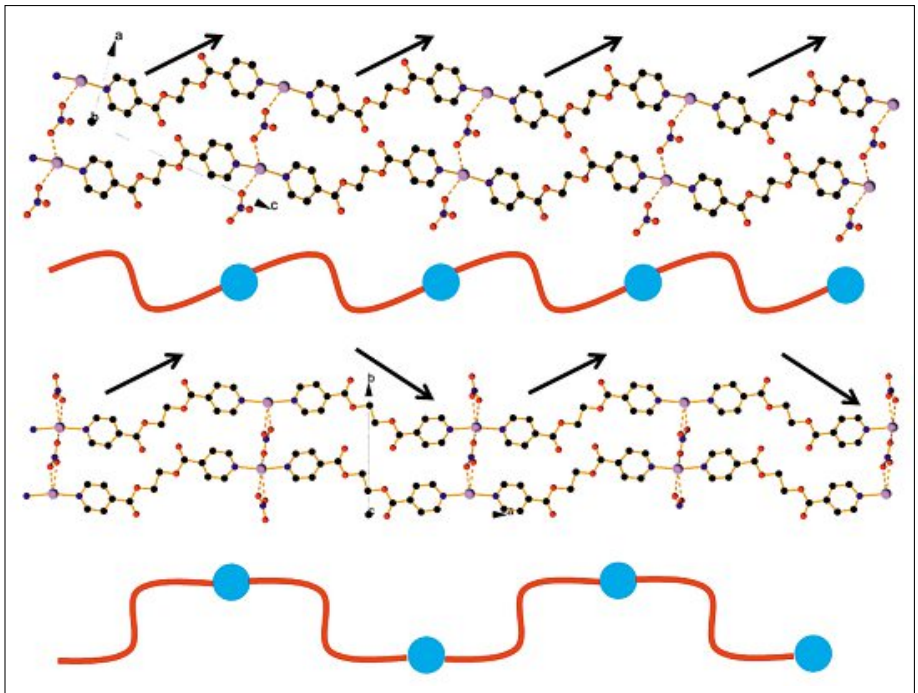

Fig. 4. 'Up-up' (top) and 'up-down' structures of the two possible deposited structures.

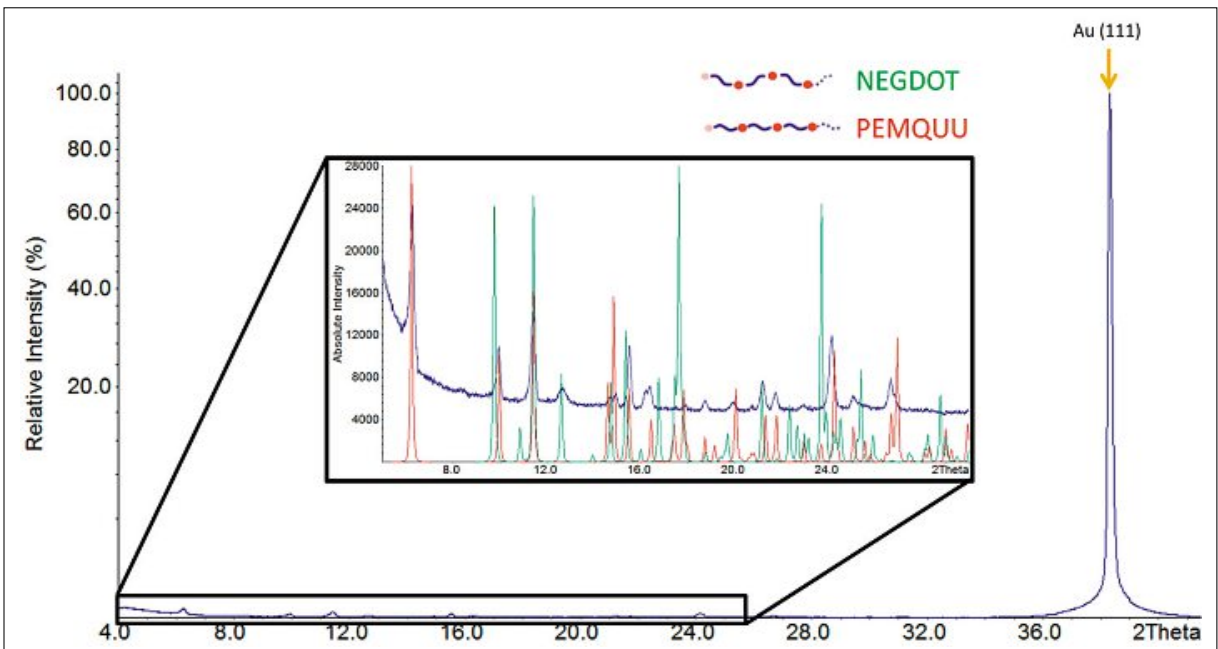

Fig. 5. Diffractogram obtained from a gold plate coated with a silver coordination polymer.

\section{Single Crystal Structure Resolution}

"The great advantage of $X$-ray analysis as a method of chemical structure analysis is its power to show totally unexpected and surprising structures with, at the same time, complete certainty."

Prof. Dorothy Crowfoot Hodgkin, Nobel lecture, 1964

Some examples are given in the following.

\section{Orientation of the Functional Group/Stereochemistry}

In this study, a bicyclic compound, 7-hydroxybicyclo[2.2.1]hept-5-ene-2,3diyl diacetate was synthesized and characterized by the classical techniques in the group of A. F. M. Kilbinger (University of Fribourg). ${ }^{[21]}$ Single crystals were obtained and submitted to our service laboratory with the request to determine the stereochemistry, i.e. the direction of the hydroxy group (Fig. 6) with respect to the alkene and ester groups of the bicycle. With this compound, methods like MS or IR spec- troscopy did not help and the determination of the position of the hydroxy group by NMR remained blurry. The single crystal X-ray structure revealed the orientation of the hydroxy group and shows the intermolecular hydrogen bonds that these hydroxyl groups are forming in the solid state.

\section{Single Crystal to Single Crystal Transformation}

Single crystal to single crystal transformation is an interesting process which can be performed by e.g. mechanical stress, ${ }^{[22]}$ vapor diffusion, ${ }^{[23]}$ photoreaction, ${ }^{[24]}$ or soaking. ${ }^{[25]}$ Such a case was observed in the Fromm group for a supramolecular single crystal obtained by crystallization of dibenzo-18-crown-6 (DB18C6) in the presence of potassium triiodide, which results in the formation of dark brown crystalline needles. ${ }^{[25]}$ The single crystal structure of this potassium compound reveals three parallel channel types built from stacked crown ether molecules, only one of which is filled with water, while the two others are partially, respectively completely filled with potassium ions and water molecules. The triiodide anions act as pillars in between these channels. Immersion of one of these single crystals into sodium hydroxide solution leads to the exchange of the potassium with sodium ions, and partial exchange of water with hydroxide ions. The number of channel types is reduced from three to two: one is filled with sodium ions and water molecules, while the other one is filled with sodium and hydroxide ions (Fig. 7). This transformation is only possible without breaking the single crystals due to the small variations in the unit cell dimensions and reveals the good ionic conductivity of the compound.

\section{Polymorphism}

$\mathrm{X}$-ray structure determination is particularly important in the case of polymorphism, manifested for example when a compound shows more than one melting point during heating, or water solubility dependent on the way of crystallization.

Polymorphism can be "[...] defined as the ability of a substance to exist as two or more crystalline phases that have different arrangements or conformations of the molecules in the crystal lattice".[26] Polymorphism is an important issue in chemistry, materials science and especially in pharmaceutical industry, and its definition can differ depending on the research area, in particular when pseudopolymorphism is concerned. A recent review on polymorphism gives more insights into this field and the tools for determining it. ${ }^{[27]}$ A recent example of polymorphism at the University of Fribourg is that of dibenzo-24-crown-8, for which two polymorphs have been crystallized. ${ }^{[28]}$ They are 


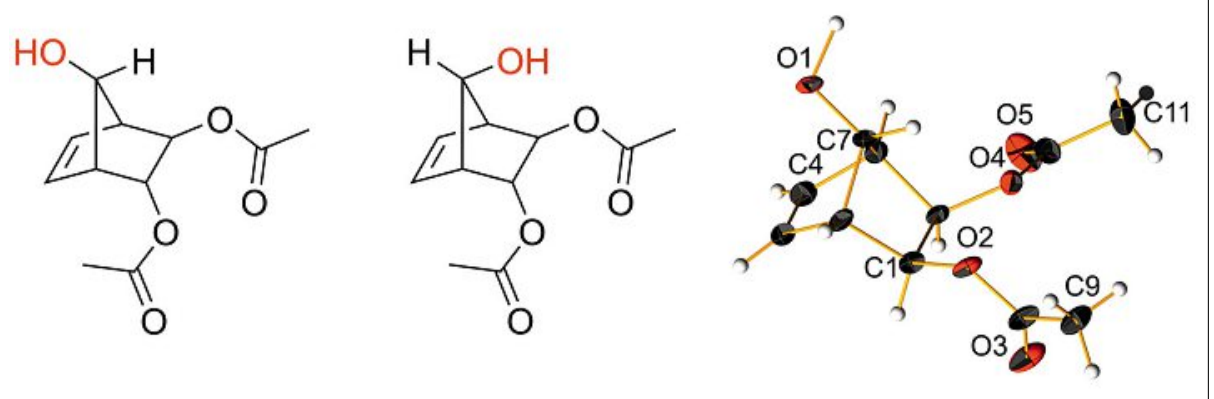

Fig. 6. The two possible configurations of the hydroxy group (left), and X-ray structure of the bicyclic compound (right).

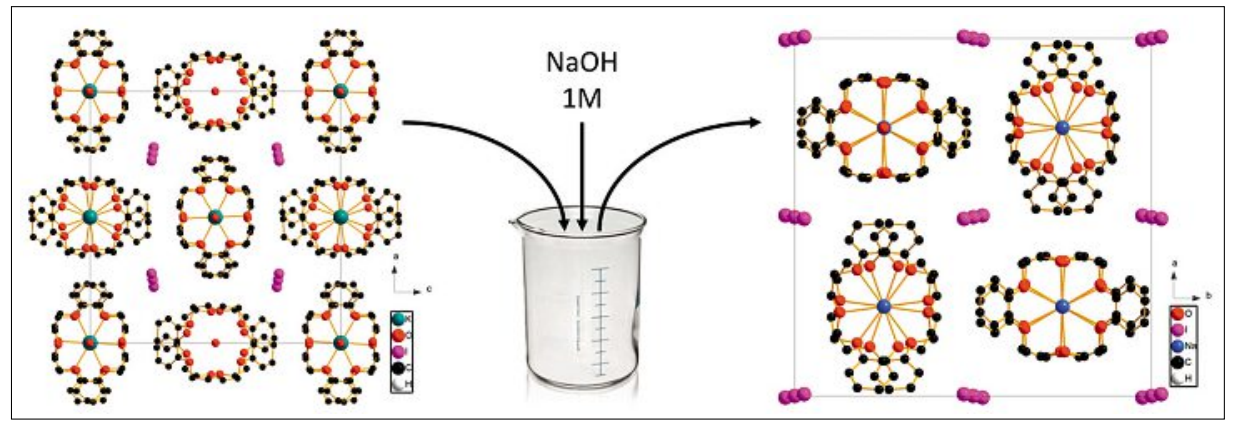

Fig. 7. Single crystal to single crystal transformation by soaking.

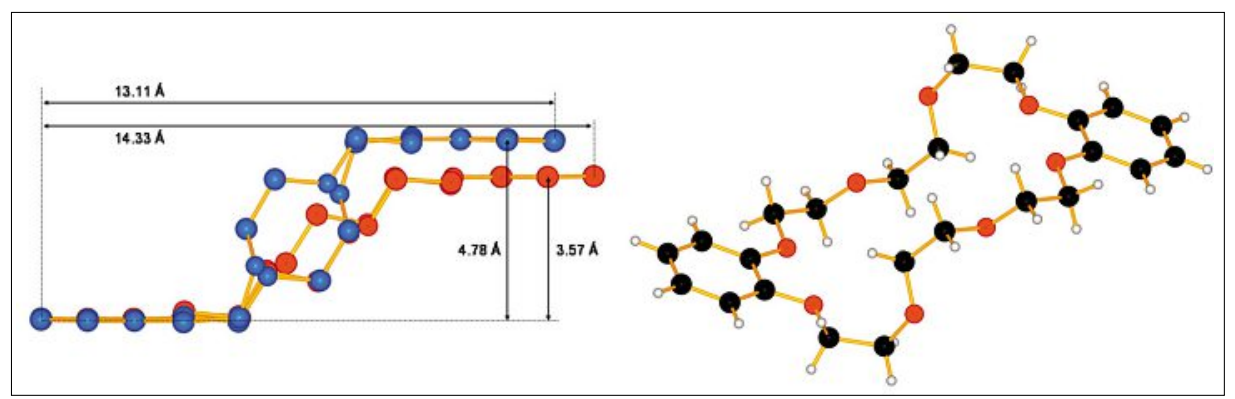

Fig. 8. (Left) Overlay of the two conformational polymorphs of dibenzo-24-crown-8, (right) structure of one polymorphic form.

called conformational polymorphs as can be easily seen if the two crystal structures are overlaid (Fig. 8).

The identification of polymorphic forms can be also performed with differential scanning calorimetry (DSC), IR or solid-state NMR spectroscopy, yet diffraction is the most efficient method to determine if two compounds are polymorphs. [27]

With these case examples, I have tried to give an overview of 'everyday' problems in university research that can be solved with state of the art diffraction facilities in classical service laboratories. In addition to that, the service crystallographers promote diffraction techniques to young researchers and teach them how to use the equipment and solve simple problems. For Master and PhD students as well as postdoctoral researchers interested in strengthening their skills in crystallography the Zurich School of Crystallography offers every two years training courses focused on single crystal diffraction (http:// www.oci.uzh.ch/group.pages/linden/zsc/).
In alternation to these courses, the PSI organizes upgrade opportunities on powder diffraction using synchrotron facilities.

\section{Acknowledgements}

The author thanks the University of Fribourg, the Fribourg Center for Nanomaterials, the Swiss National Science Foundation and all colleagues of the University of Fribourg for the authorization to use the unpublished data.

Received: January 25, 2014

[1] W. Friedrich, P. Knipping, M. Laue, Ann. Phys. 1913, 346, 971

[2] W. L. Bragg, Proc. Royal Soc. A 1913, 89, 248.

[3] W. H. Bragg, W. L. Bragg, Proc. Royal Soc. A 1913, 89, 277.

[4] Thomson Reuters, Web of Science (search carried out for 2012, respectively 1992, in November 2013).

[5] a) L. Antonov, V. Kurteva, A. Crochet, L. Mirolo, K. M. Fromm, S. Angelova, Dyes Pigm. 2012, 92, 714; b) Y. Cheremond, A. Crochet, K. M. Fromm, Eur. J. Inorg. Chem. 2012, 2012, 2725 ; c) A. Crochet, I. Alimi, C. G. Bochet, K. M. Fromm, Acta Crystallogr., Sect. E: Struct. Rep. Online 2013, 69, o339; d) A. Crochet, K. M. Fromm, Polyhedron 2013, 52, 610; e) A.
A. Nagarkar, A. Crochet, K. M. Fromm, A. F. M. Kilbinger, Macromol. 2012, 45, 4447; f) A. Trachsel, B. Buchs, G. Godin, A. Crochet, K. M. Fromm, A. Herrmann, Eur. J. Org. Chem. 2012, 2837, S2837/1-S2837/37.

[6] a) E. C. Constable, C. E. Housecroft, S. Vujovic, J. A. Zampese, A. Crochet, S. R. Batten, CrystEngComm 2013, 15, 10068; b) A. K. Inge, H. Fahlquist, T. Willhammar, Y. Huang, L. B. McCusker, X. Zou, J. Appl. Crystallogr. 2013, 46, 1094; c) D. Xie, L. B. McCusker, C. Baerlocher, S. I. Zones, W. Wan, X. Zou, J. Am. Chem. Soc. 2013, 135, 10519; d) R. Cerny, P. Schouwink, Y. Sadikin, K. Stare, L. Smrcok, B. Richter, T. R. Jensen, Inorg. Chem. 2013, 52, 9941.

[7] a) A. Neels, L. d. A. Vieira, M. Döbeli, A. Dommann, J. Herrán, F. Neff, B. Widrig, H. Brändle, J. Ramm, Adv. Eng. Mater. 2011, 13, 87; b) D. Gopi, J. Indira, L. Kavitha, J. M. F. Ferreira, J. Appl. Electrochem. 2013, 43, 331; c) A. Satapathy, H. Sutar, S. C. Mishra, S. K. Sahoo, J. Am. Chem. Sci. 2013, 3, 151.

[8] J. Charmet, J. Bitterli, O. Sereda, M. Liley, P. Renaud, H. Keppner, J. Microelectromech. Syst. 2013, 22, 855.

[9] G. Gururajan, H. Shan, G. Lickfield, A. A. Ogale, Polym. Eng. Sci. 2008, 48, 1487.

[10] CCDC, CSD (Cambridge Structural Database) (www.ccdc.cam.ac.uk).

[11] Crystallography Open Database, Crystallography Open Database (www. crystallography.net).

[12] FIZ Karlsruhe, ICSD - Inorganic Crystal Structure Database (www.fiz-karlsruhe.de).

[13] R. Downs, M. Hall-Wallace, Am. Mineralogist 2003, 88, 247.

[14] A. Devaux, A. Crochet, K. M. Fromm, P. Belser, unpublished results.

[15] C. Baerlocher, L. B. McCusker, 'Database of Zeolite Structures', www.iza-structures.org/ databases/

[16] A. Z. Ruiz, D. Brühwiler, T. Ban, G. Calzaferri, Monatshefte für Chemie 2005, 136, 77.

[17] A. Y. Robin, J. L. Sague Doimeadios, A. Neels, T. Vig Slenters, K. M. Fromm, Inorg. Chim. Acta 2007, 360, 212.

[18] a) I. Chevrier, J. L. Sague, P. S. Brunetto, N. Khanna, Z. Rajacic, K. M. Fromm, Dalton Trans. 2013, 42, 217; b) S. Eckhardt, P. S. Brunetto, J. Gagnon, M. Priebe, B. Giese, K. M. Fromm, Chem. Rev. 2013, 113, 4708; c) K. M. Fromm, Nature Chem. 2011, 3, 178. d) K. M. Fromm, Appl. Organomet. Chem. 2013, 27, 683; e) O. Gordon, T. V. Slenters, P. S. Brunetto, A. E. Villaruz, D. E. Sturdevant, M. Otto, R. Landmann, K. M. Fromm, Antimicrob. Agents Chemother. 2010, 54, 4208.

[19] P. S. Brunetto, K. M. Fromm, Chimia 2008, 62, 249.

[20] a) A. Neels, O. Sereda, T. Bandi, X. Maeder, Chimia 2014, 68, 14; b) L. B. McCusker, C. Baerlocher, Chimia 2014, 68, 19.

[21] A. A. Nagarkar, A. Crochet, K. M. Fromm, A. F. M. Kilbinger, unpublished result.

[22] H. Ito, M. Muromoto, S. Kurenuma, S. Ishizaka, N. Kitamura, H. Sato, T. Seki, Nat. Commun. 2013, 4, 3009/1.

[23] L. R. Nassimbeni, H. Su, CrystEngComm 2013, 15, 7396.

[24] G. K. Kole, A. M. P. Peedikakkal, B. M. F. Toh, J. J. Vittal, Chem. Eur. J. 2013, 19, 3962.

[25] C. D. Assouma, A. Crochet, Y. Chérémond, B. Giese, K. M. Fromm, Angew. Chem. Int. Ed. 2013, 52, 4682

[26] R. Purohit, P. Venugopalan, Reson 2009, 14, 882.

[27] J.-P. Brog, C.-L. Chanez, A. Crochet, K. M. Fromm, RSC Adv. 2013, 3, 16905.

[28] A. Crochet, E. Kottelat, A. Fleury, M. Neuburger, K. M. Fromm, Z. Anorg. Allg. Chem. 2011, 637, 672. 